\title{
TECNOLOGIA DA INFORMAÇÃO E COMUNICAÇÃO: NOVAS TENDÊNCIAS DO ENSINO NA EDUCAÇÃO FÍSICA
}

\author{
INFORMATION AND COMMUNICATION TECHNOLOGY: NEW TEACHING TRENDS IN \\ PHYSICAL EDUCATION \\ TECNOLOGÍA DE INFORMACIÓN Y COMUNICACIÓN: NUEVAS TENDENCIAS DE ENSEÑANZA \\ EN EDUCACIÓN FÍSICA
}

\author{
Tiago Cesar Fuzaro \\ Mestre em ciências pela Universidade de São Pulo, USP. \\ E-mail: fuzarounicamp@yahoo.com.br. \\ Orcid: https://orcid.org/0000-0003-3228-0767
}

\author{
Eduardo Ferro dos Santos \\ Pós -Doutor em Inovação e Tecnologias do Ensino, Professor da Universidade de São Paulo. \\ E-mail: eduardo.ferro@usp.br. \\ Orcid: : https://orcid.org/0000-0002-1212-6714
}

\author{
Marco Aurélio Alvarenga Monteiro \\ Doutor em Educação, Professor da Universidade do Estado de São Paulo. \\ E-mail: marco.aurelio.feg@gmail.com. \\ Orcid: https://orcid.org/0000-0002-4426-1638
}

\begin{abstract}
RESUMO
Neste estudo são expostas informações de abrangência internacional a respeito das adaptações que vem ocorrendo na área de Educação Física (EF) e o atrelar inexorável com as Tecnologias da Informação e Comunicação (TICS) referentes às pesquisas científicas dos últimos 14 anos. O objetivo desse artigo foi inquirir as oportunidades e tendências de pesquisa para área da Educação Física (EF). Para tal recorreu-se a uma pesquisa bibliométrica nas plataformas Web of Science e Scopus, assim como a leitura cuidadosa e investigativa dos artigos mais citados segundo essas bases de dados. As três tendências principais para futuras pesquisas relacionando as TICS e a EF foram identificadas na análise dos gaps dos artigos mais citados, destacando-se entre elas três grandes grupos: a criação e padronização do(s) material(is) e método (s) "CPMM"; a pesquisa com foco no(s) aluno(s) "FNA"; e a investigação com um maior foco no(s) professor(es) "FNP" Desta forma sugere-se que as novas pesquisas sigam tais vertentes de estudo, enaltecendo de forma próspera a área científica nas múltiplas relações possíveis entre as TICs e EF.
\end{abstract}

Palavras-chave: Tecnologia da informação e comunicação. Ensino. Educação física.

\section{ABSTRACT}

This study exposes information of international scope regarding the adaptations that have been occurring in the Physical Education (PE) area added to the inexorable link with Information and Communication Technologies (ICTs) related to the scientific research of the last 14 years. The purpose of this article was to inquire about research opportunities and trends in the Physical Education field. For this purpose, a bibliometric research was used in the Web of Science and Scopus platforms, as well as the careful and investigative reading of the most cited articles according to these databases. The three main trends for 
future research relating ICTs and PE were identified in the gap analysis of the most cited articles, with three major groups standing out: the creation and standardization of the material (s) and method (s) "CSMM"; research focusing on the student (s) "FOS"; and research with a greater focus on the teacher (s) "FOT". In this way, it is suggested that the new researches follow such strands of study, successfully extolling the scientific area in the multiple possible relationships between ICTs and PE.

Keywords: Information and communication technology. Teaching. Physical education.

\section{RESUMEN}

En este estudio, se exponen informaciones de alcance internacional con respecto a las adaptaciones que se han estado produciendo en el área de Educación Física (EF) y el vínculo inexorable con las Tecnologías de la Información y la Comunicación (TIC) en lo que se refiere a la investigación científica de los últimos 14 años. El propósito de este artículo fue indagar sobre las oportunidades de investigación y tendencias en el campo de la educación física. Para este propósito, se utilizó una investigación bibliométrica en las plataformas Web of Science y Scopus, así como la lectura cuidadosa e investigativa de los artículos más citados de acuerdo con estas bases de datos. Las tres tendencias principales para futuras investigaciones relacionadas con las TIC y la EF se identificaron en el análisis de las lagunas de los artículos más citados, destacando tres grupos principales: la creación y estandarización de los materiales y métodos. "CEMM"; investigación centrada en el / los estudiante (s) "ICE"; e investigación con un mayor enfoque en el maestro (s) "ICM". Por lo tanto, se sugiere que las nuevas investigaciones sigan tales líneas de estudio, exaltando con éxito el área científica en las múltiples relaciones posibles entre las TICs y EF.

Palabras-clave: Tecnología de la información y la comunicación. Enseñanza. Educación física.

\section{INTRODUÇÃO}

A tecnologia vem mudando a forma com que os humanos se relacionam, culminando claramente em uma alteração visível na comunicação, quer seja essa no âmbito da esfera profissional, emocional, familiar ou do entretenimento etc. É inegável a contribuição crescente que a tecnologia tem feito para nossa sociedade atual quando refletimos sobre os aspectos de ensino e de aprendizado.

No que tange a área da educação, as TICs somam com a criação de novas formas de receber e passar o conhecimento, concedendo assim desde novos ambientes de aprendizagem tais como: os Massive Open Online Courses (MOOCs) e as plataformas de ensino a distância (EAD), até as novas metodologias ativas que fazem a utilização de games on-lines e blogs que proporcionam um aprendizado mais significativos e prazeroso.

De um lado o avanço descomunal da tecnologia high-tec impulsionando a instrução e desenvolvimento de novos estudantes, de outro as barreiras de caráter político, econômico e social que muitas vezes freiam a evolução das novas perspectivas educativas. 
Não existe uma definição única e imutável do significado de Tecnologia da Informação e Comunicação (TIC). De forma ampla TIC pode ser definida como todos os meios técnicos usados para abordar a informação e ajudar na comunicação. Outros nomes podem evidenciar o uso das TICs, por exemplo E-learning, que segundo Davis, Kemis e Johnson (2003) é o nome de um conjunto de ferramentas que objetiva conectar o ensino com os avanços tecnológicos, de certa forma aperfeiçoando o ensino e/ou propagar da informação, podendo assim inquirir que tal reduz as fronteiras, quer sejam elas em uma esfera local, temporal ou cultural.

TIC é a sigla referente a Tecnologia de Informação e Comunicação, conquanto nas pesquisas de base de dados internacionais, os documentos relacionados a essa temática usam a sigla ICT, correlata ao nome grafado na Língua Inglesa "Information and Communication Technology". Todavia nesse texto usaremos a sigla TIC, ou então TICs fazendo referências as tecnologias "plural”.

O dicionário Oxford online descreve as TIC como sendo uma abreviação de "tecnologia da informação e comunicação", ou seja, "o estudo do uso de computadores, da Internet, do vídeo e de outras tecnologias como assunto na escola" (OXFORD on line).

A vista dessas afirmações e esclarecimento quanto as TICs, questiona-se nesse artigo, como estão os estudos na área da Educação Física (EF) que interagem com as tecnologias atuais. Uma área tão palpável e corpórea como a EF teria alguma conexão com as TICs? Nesse artigo buscou-se esquadrinhar as novas tendências de pesquisa nessa área inter-relacionando-as, e para tal foi necessário estudar o que tem sido publicado com relação a essas temáticas nos últimos anos.

\section{Contextualização das TICs no âmbito do ensino}

A sociedade atual está globalizada, cada vez mais apoiada e dependente da tecnologia para estabelecer relacionamentos ou obter dados, quer sejam eles no foro social, profissional ou educacional. Logo, nos dias de hoje se faz notável o uso das TICs no ensino, quer sejam elas na esfera formal ou informal do aprendizado, dentro ou fora das salas de aulas, em suma, muitos tem aprendido com ajuda das tecnologias.

A utilização das tecnológicas e suas muitas formas de expressão no ensino não podem ser esquecidas ou ignoradas, caso isso ocorra existe a possibilidade de se 
construir uma formação falha para aquele que estão sendo moldado (SUZUKI; RAMPAZZO, 2009).

Para que tal falha não ocorra no cenário da educação, as TICs somam com como possibilidades para que haja variedade na forma de transmitir os conteúdos, informações, dados, os quais podem se transformar em conhecimento "informação para tomada de decisão" (SEMIDÃO, 2014). No final da década de sessenta, Ausubel et al. (1968), sugeriram por meio da teoria construtivista que para transformar informação em conhecimento, os alunos têm que torná-lo próprio, ganhar o controle dele, além de construir o conhecimento subjetivo.

Com intuito de tornar essa possibilidade viável, o governo do estado e o governo federal brasileiro amparam a inclusão gradual das tecnologias nas escolas, visto que o Programa Nacional de Tecnologia Educacional - Proinfo foi criado com esse desígnio pelo Decreto n. 6.300 artigo $1^{\circ}$. Com essa base legal, a prática pedagógica tem a possibilidade de ser mais atualizada e flexível, e os docentes podem dispor de uma estrutura diferenciada para buscar a melhor forma para ensinar os alunos brasileiros (BRASIL, 2007).

Por meio de investigação os autores Basadre, Nunez e Paton (2015) concluem que os avanços tecnológicos devem ser usados como ferramentas para melhorar e complementam as diferentes áreas de um currículo, por tanto, faz-se necessário uma reflexão sobre o quanto as TICs estão sendo utilizadas atualmente, e se as mesmas estão sendo pinçadas para utilização por livre arbítrio dos professores, ou se já fazem parte da grade curricular e recursos de algumas instituições. De fato, este não é o norte do presente artigo, mas passar por essa temática sem comentar sobre tal seria no mínimo um descaso, visto a ligação íntima com o assunto que está sendo pensado.

Quanto o panorama da aquisição do conhecimento, Jonassen (1999), aborda que as tecnologias podem cooperar como auxiliadoras em uma aprendizagem ativa, assim como já foi relatado que as TICs podem induzir uma maior reflexão dos alunos sobre o conteúdo dado e propiciar um maior compartilhamento do aprendizado com outras pessoas (SUSMAN, 1998).

\section{Relação as TICs e a EF}


No que diz respeito a relação entre as TICs e a EF, a colocação de Hartveld, Hegarty e Blurton (1996), foram bem categóricos quando notaram que pouco foi estudado sobre o uso das tecnologias no desenvolvimento das capacidades corporais nas aulas de EF, todavia, outros autores ressaltam que na atualidade, as aplicações de multimídia no âmbito educacional têm sido muito usada no ensino superior (KIRKWOOD et al., 2002; WIKSTEN; SPANJER; LA MASTER, 2002; ANTONIOU et al., 2003).

A EF e sua subjetividade pedagógica, a qual está intimamente atrelada às práticas corporais, possui dificuldades ímpares na tentativa de perceber, entender e utilizar os recursos tecnológicos nas aulas. Somado a isso, a maioria das aulas, de alguma forma, estão associadas as restrições quanto: a infraestrutura física e material disponibilizado; o suporte técnico para o uso adequado dos recursos; assim como as concepções dos professores sobre a relação da própria EF e as novas tecnologias educativas (FERREIRA; BENDRATH; BASEI, 2016). Com relação a essa última restrição citada, discorreremos um pouco mais adiante no texto.

Exemplificando um pouco sobre o auxílio das estruturas físicas no ensino com as TICs, pode-se perceber na conjuntura atual em sala de aula, a relevância dos vídeos e das imagens digitais para o processo de aprendizagem, os quais assumem funções facilitadoras, que ajudam a assimilar tanto conceitos, como habilidades motoras (ANDERSON; MIKAT; MARINEZ, 2001; MOHNSEN, 2008).

As TICs, podem colaborar muito na EF, pois esta ferramenta possibilita que os alunos visualizem movimentos, ouçam, ilustrem e até criem tais movimentos sem executar a atividade prática em si (OLIVEIRA, 2010). Como exemplo, pode-se citar uma rústica filmagem de um salto vertical feita por um celular, que após filmado pode ser transferido e salvo para um computador, e com a utilização de algum software atual, tal como o TRACKER, o salto gravado pode ser analisado. Depois de alguns minutos, na tela de um notebook, propiciará aos alunos a visualização do movimento em diversas velocidades, obter a altura saltada calculada, gerar um gráfico da velocidade entre outros recursos.

Nesta mesma linha de pensamento, infere-se que a utilização de um simples DVD ou da internet em si, pode propiciar a apreciação de esportes ou práticas corporais como a canoagem, tecido acrobáticos ou o kitesurf, que são na maioria das vezes inviáveis em escolas e universidade (POLATO, 2009). 


\section{Contextualização TICs e os professores de EF}

Para educar formando cidadãos e profissionais reflexivos é imprescindível que aqueles que trabalham ministrando aulas estejam atualizados com tais mudanças, pois educar para as novas tecnologias é formar as mais amplas faculdades, desde a memorização até o julgamento baseado em um senso mais crítico (PERRENOUD, 2000).

As TICs inseridas no ensino podem otimizar a aprendizagem de quem está aprendendo. Cabe aos professores conhecerem o potencial dessas tecnologias. Em uma outra ótica, os professores também devem ser estimulados para que tenham contato com as TICs, assim tendo a viabilidade de produzir um ambiente mais propicio para uma aprendizagem ativa e independente aos alunos (SMEETS, 2005).

Os docentes e seus múltiplos prismas pedagógicos tem como diretriz principal a ideia de que essas tecnologias podem sim cooperar nas aulas gerando um ganho de conhecimento para os alunos, consequentemente, as TICS tornam-se valorosas nas salas de aula (HIGGINS; MOSELEY, 2001; NIEDERHAUSER; STODDART, 2001).

\section{Problema atual}

Guardia (2002), assimila que a TIC é uma ferramenta capaz de incorporar novas formas de conhecimento entre professores e alunos na perspectiva da EF. No entanto, os professores de EF tem o desafio de incorporar ao seu método de ensino, os recursos das TICs, mesmo sem que eles tenham tido a oportunidade de ter aprendido sobre tais na faculdade que cursaram (GARCÍA; SÁNCHEZ, 2014).

Um estudo grandioso com mais de 500 escolas foi feito por toda a Inglaterra relacionando as TICs e os professores de EF. A pesquisa de Thomas e Stratton (2006), relata algumas dificuldades em usar as TICs no âmbito da EF. No artigo, o tempo foi visto como a principal barreira no uso das TICS nas aulas de EF, visto que a maior parte dos professores necessitam de bastante tempo para configurar os equipamentos tecnológicos. Além disso, o tempo não é somente para preparar o recurso tecnológico em aula, mas também para que os professores possam produzir esses recursos. 
A falta de tempo também foi vista como uma barreira no uso das TICs quando foi feita a relação com o tempo gasto para treinamentos das TICs e seus respectivos problemas e falhas, visto que é necessário saber lidar com tais, caso ocorram durante as apresentação de aula, assim como entender sobre os equipamentos e softwares utilizados. Por esses e muitos outros motivos, inúmeros educadores físicos não vem desenvolvendo uma ligação do tema tecnológico desde a perspectiva inicial nas escolas (GARCÍA; SÁNCHEZ, 2014).

Um outro aspecto dificultoso no uso das TICS na EF está correlacionado com a eficiência da ferramenta utilizada. No estudo de Papastergiou, Gerodimos e Antoniou (2011) os autores optaram pelo uso dos "bloggings educacionais" para envolver os 70 alunos da graduação do Departamento de Educação Física e Ciências do Esporte (DPESS) da Universidade de Tessália na Grécia, em uma produção ativa de conteúdos multimídia e interações sociais nas aulas de basquete. A experiencia evidenciou que o grupo que utilizava as TICs, neste caso o blog, não apresentou maiores níveis de conhecimento ou habilidades específicas de basquete do que o grupo que não usou o blog. Neste estudo, a participação na atividade de blogging foi igualmente ineficaz tal como a utilização do "website".

Porém, esta conclusão difere com os resultados de duas outras publicações científicas feitas também com estudantes de graduação, as quais pautaram-se em medidas objetivas de aprendizagem dos alunos com a utilização dos blogs com fim educacional, desencadeando uma resposta positiva nas notas dos alunos (EL TANTAWI, 2008).

De fato, a utilização das TICs depende de muitos fatores e processos para atender determinados objetivos no ramo do ensino. As TICs não podem ser entendidas como uma “ferramenta pedagógica mágica” para sanar problemas de toda e qualquer circunstância na esfera que cerne o aprendizado.

Uma das importâncias da TIC é que tal pode gerar um progresso educacional integral e atualizado. A acessibilidade a tais tecnologias se torna cada vez maior. A relação entre as instituições educacionais e a formação de professores com as TICs são muito valorosas para todo esse processo, os problemas como falta de tempo e a constatabilidade ou não da eficiencia dessas ferramentas também alavancam esse estudo para uma pesquisa bibliométrica aprofundada, com o intuito de buscar uma visão mais 
abrangente dessa temática buscando os possíveis gaps de forma a propiciar uma linha de orientação para pesquisas vindouras.

\section{Métodologia}

A magnitude de uma pesquisa pode ser mensurada pelo número de publicações acadêmicas que aborda o tema e pelas múltiplas oportunidades de trabalhos futuros sugeridos em tais publicações. Para abordar novos desafios no campo do ensino e aprendizado da EF, realizou-se neste trabalho uma análise bibliométrica quantitativa de documentos publicados on-line. Assim, pode-se compreender um pouco mais das diferentes vertentes relacionando a área de EF e as Tecnologias de Informação e Comunicação (TICs ou ICTs - sigla grafada em inglês por motivos já citados).

As pesquisas acadêmicas podem nortear a utilização das TICS nas mais variadas áreas, além de contribuir de forma expressiva e prática no ensino. A fim de identificar em que direção a literatura se desenvolve e de buscar oportunidades de pesquisa em relação a este tema, foi realizada uma análise bibliométrica do assunto.

Logo, a pesquisa bibliométrica serve como alicerce para a criação de novos conhecimentos. Dentre os possíveis objetivos das pesquisas bibliométricas, temos: o levantamento dos pareceres de outros pesquisadores sobre o estudo a ser pesquisado, a identificação de lacunas investigativas, a utilização, como material de estudo, dos trabalhos desenvolvidos por outros pesquisadores, dentre outros (MEDEIROS et al., 2015).

A bibliometria é a prática da ciência que compreende o uso de instrumentos matemáticos e estatísticos com o objetivo de analisar e medir a literatura em um determinado campo de conhecimento (HUANG et al., 2016). Compreende à análise quantitativa de dados para investigar quais são suas propriedades e como se comporta a evolução das informações no meio científico (FERREIRA et al., 2014). É uma ferramenta importante para investigar o campo de pesquisa do ensino atrelado com a utilização das TICs para identificar sua origem e evolução.

Como fora anunciado anteriormente, um dos objetivos deste trabalho é identificar qual é o estado da arte da literatura, quais são as oportunidades e tendências da pesquisa no assunto. Para tal, foram mapeados a produção científica considerando os anos de 
publicação, autores, área de pesquisa assim como os países mais relevantes e os journals mais utilizados, conjeturando assim a evolução da produção de literatura em um determinado período de tempo (GOMEZ-JAUREGUI et al., 2014).

Foi realizada uma análise bibliométrica sobre TICs e a área de EF, buscando autores e publicações relevantes sobre o tema. Para isso, foi selecionada as bases de dados "Web of Science e Scopus". Estas bases de dados foram escolhidas por conta da grande relevância e destaque que elas têm no âmbito científico nacional e internacional, além de propiciarem uma pesquisa refinada e objetiva quanto ao tema.

Para a busca de trabalhos nas bases de dados, foram utilizadas as palavras chave “Physical Education”, "Sport”, “ICT” e "Information and Communication Technology". Tais palavras deveriam estar contidas sempre nos títulos dos trabalhos. A busca se deu por meio de 4 etapas cruzando as palavras na seguinte ordem proposta: primeira pesquisa usando as palavras Physical Education e ICT; segunda pesquisa usando as palavras Physical Education e Information and Communication Technology; terceira com Sport e ICT e por último relacionando as palavras Sport e Information and Communication Technology.

Um dos filtros utilizados na bibliometria em ambas plataformas foi: a restrição das publicações para artigos, publicações em eventos, capítulos de livros e revisões com as palavras supracitadas contidas no título. Este filtro foi adotado para aumentar a precisão dos resultados, entendendo-se que eles permitem uma identificação mais precisa e confiável das lacunas científicas relacionadas ao tema.

Outro filtro aplicado foi a delimitação de trabalhos publicados nos anos de 2006 a 2019 para melhor compreensão do estado da arte e a identificação de lacunas científicas atuais para promover e orientar novos estudos sobre o tema.

Por meio da análise bibliométrica a literatura sobre a relação das TICs e a EF foi explorada e analisada. Os parâmetros bibliométricos utilizados neste estudo foram: os números de artigos publicados e os números de citações recebidas, considerando os anos de tais publicações, os principais autores, as áreas de pesquisa que abordam esse tema assim como os países mais relevantes e os journals mais utilizados para divulgação de tal. Esta bibliometria mostra a evolução da literatura ao longo dos anos e de forma mais focal nos últimos 14 anos a respeito desse assunto.

Este estudo identificou que tanto a plataforma Web of Science como a Scopus apontam informações similares sobre o tema. Apenas algumas diferenças foram notadas 
em ambas bibliometrias, visto que são plataformas que possuem formatações diferentes, mas nenhuma informação se mostrou antitética a outra, ou foi encontrado algum dado que invalida as informações que foram encontradas, pelo contrário, elas se somam e se reafirmam, ou seja, se complementam.

Quanto a progressão das publicações, ambas plataformas mostraram que o número de artigos cresceu muito de 2006 para 2019, havendo uma oscilação bem perceptível quanto ao número de documentos escritos durante esse período.

Figura 1: Documentos produzidos por ano usando as palavras-chave selecionadas nas bases de dados Web of Science e Scopus

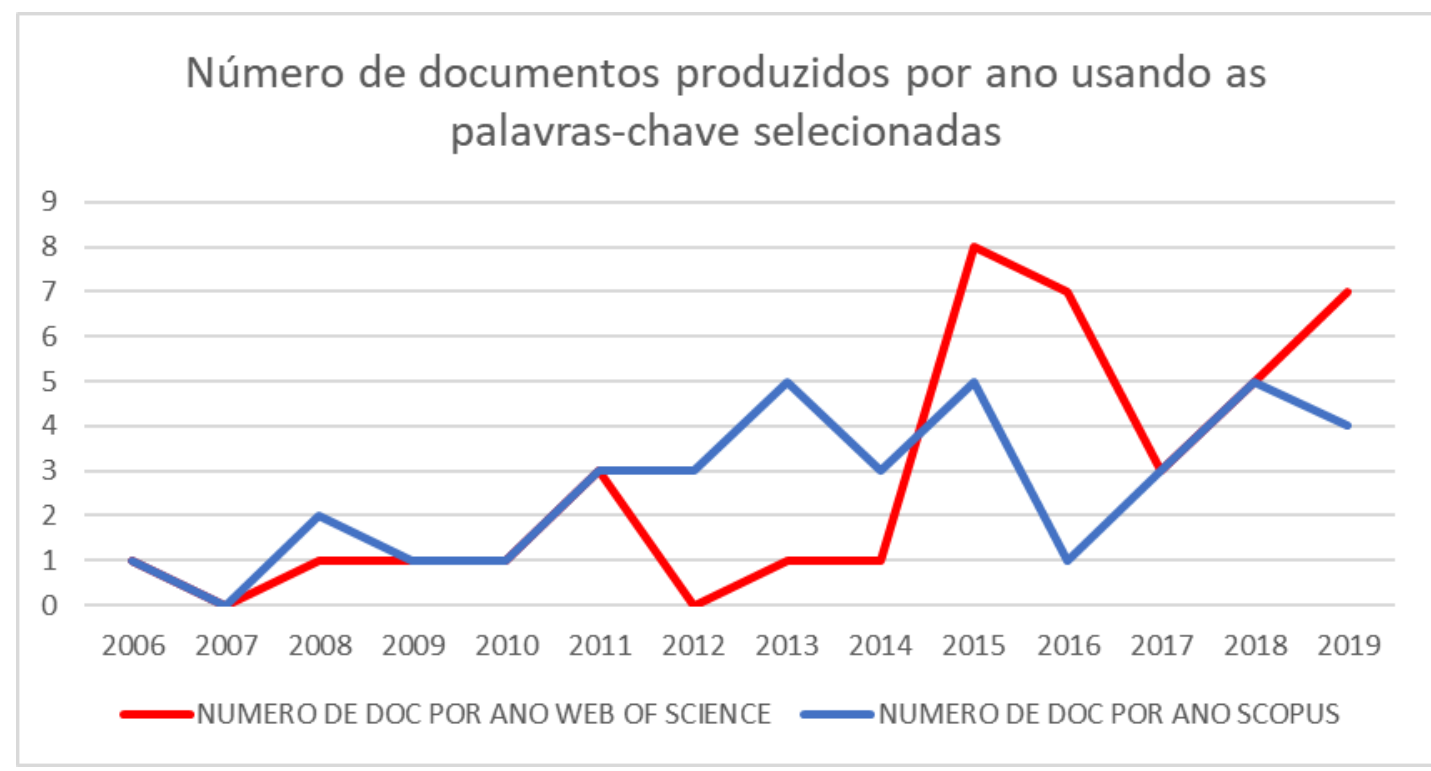

Fonte: Próprio Autor (2020)

Com relação as áreas de pesquisa, o campo da Educação foi o mais investigado, seguido das áreas de Computação, Profissões da Saúde, Arte e Humanidade e Ciências do Esporte.

Tabela 1: Ordem das áreas onde houve mais publicação de documentos produzidos durante os últimos 14 anos usando as palavras chave selecionadas

$\begin{array}{ll}1^{\mathrm{a}} & \text { EDUCAÇÃO } \\ 2^{\mathrm{a}} & \text { COMPUTAÇÃO }\end{array}$




$$
\begin{array}{c|c}
3^{\mathrm{a}} & \text { PROFISSÕES DA SAÚDE } \\
4^{\mathrm{a}} & \text { ARTE E HUMANIDADE } \\
\hline 5^{\mathrm{a}} & \text { CIÊNCIAS DO ESPORTE }
\end{array}
$$

Fonte: Próprio Autor (2020)

Quanto aos países, a Espanha, Inglaterra, Grécia, França, Canadá e Irã são os países onde mais pesquisas são publicadas sobre esse tema.

Figura 2 - Documentos relacionados ao tema publicados por países entre 20062019

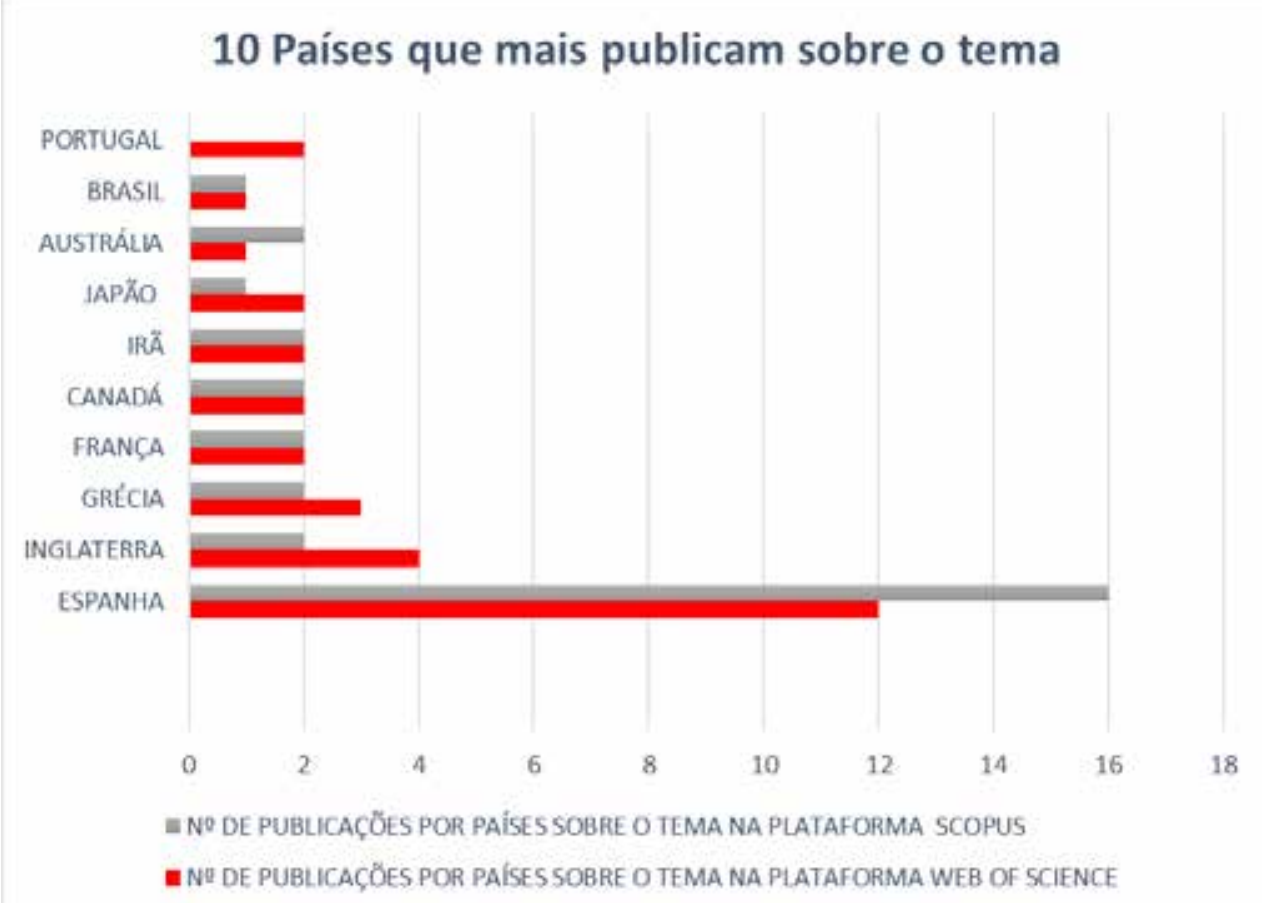

Fonte: Próprio Autor (2020)

Os journals que publicam artigos relacionando as TICs e EF são bem variados, só na plataforma da Web of Science somam o total de mais de 27 diferentes journals, não obstante a COMPUTERS AND EDUCATION é o mais requisitado quando somadas as duas plataformas. Os autores seguem na mesma perspectiva, muitos trabalhos publicados por autores diferentes, contudo a pesquisa indica que Papastergiou foi a autora que mais publicou. Ela possui 3 publicações sobre esse tema quando associadas as duas plataformas. Quando analisado os trabalhos mais citados, ela ocupa a segunda colocação 
com o trabalho "Multimedia blogging in physical education: Effects on student knowledge and ICT self-efficacy" (PAPASTERGIOU; GERODIMOS; ANTONIOU, 2011) assim como a quarta colocação com o artigo “Enhancing Physical Education and Sport Science students' self-efficacy and attitudes regarding Information and Communication Technologies through a computer literacy course"(PAPASTERGIOU, 2010) angariando com os dois trabalhos até a presente data um total de 56 citações na Web os Science e 80 vezes na Scopus.

\section{Resultados}

A partir da leitura dos 6 artigos mais citados é possível identificar oportunidades de pesquisa a serem exploradas, estes trabalhos são evidenciados no Quadro 1. Além disso, para avaliar melhor as possibilidades de pesquisa, os 6 artigos mais citados publicados entre 2006 e 2019 entre a plataforma Web of Science e Scopus, foram analisados, extraindo deles os gaps da literatura científica que eles evidenciaram.

O processo de identificação de gaps científicos sobre o tema foi conduzido para encontrar sugestões dadas pelos autores quanto aos avanços que são necessários ou cabíveis em cada um dos artigos.

No Quadro 1 a seguir, para facilitar a leitura e comparação os artigos foram organizados evidenciando o título do artigo, os autores, a revista a qual o artigo foi publicado, o ano de publicação, as lacunas encontradas durante o estudo de cada um deles além dos gaps, sendo estes as oportunidades para futuros estudos.

Em muitas das escritas, os gaps são identificados como um viés ainda não pesquisado, ou que poderia ser estudado com mais aprofundado ou abrangência, desta forma, lançando luz sobre o tema da interferência das TICs e EF.

Quadro 1 - Quadro com os gaps científicos

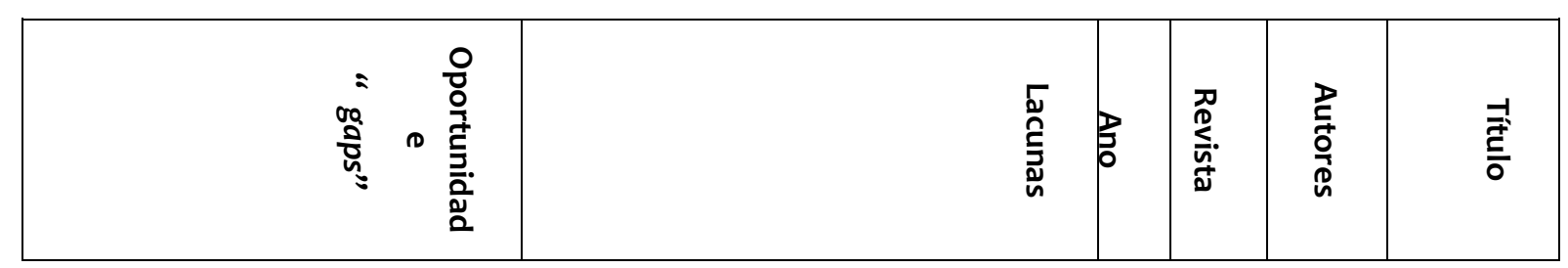




\begin{tabular}{|c|c|c|c|c|c|c|c|c|c|}
\hline 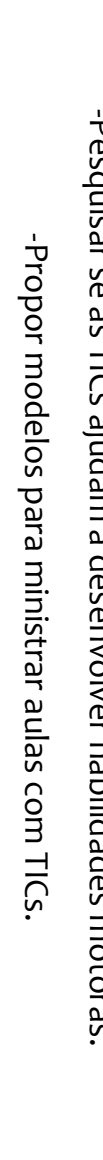 & 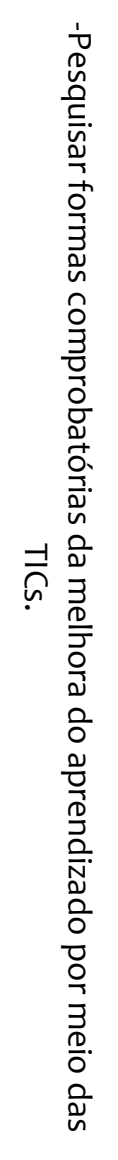 & 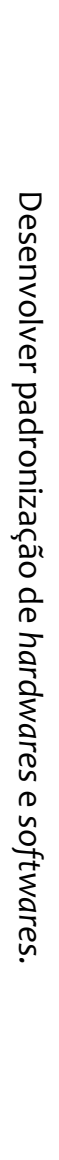 & 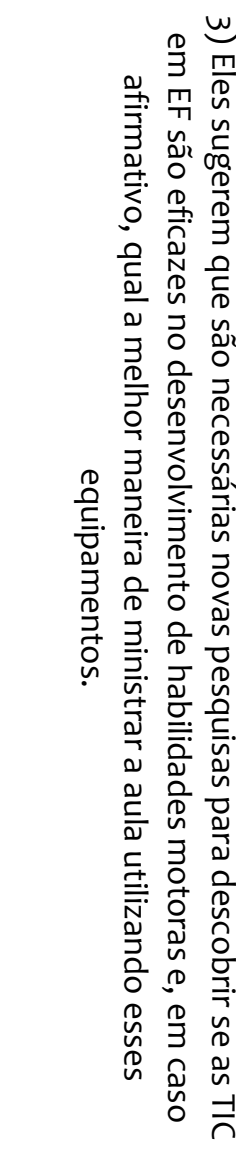 & 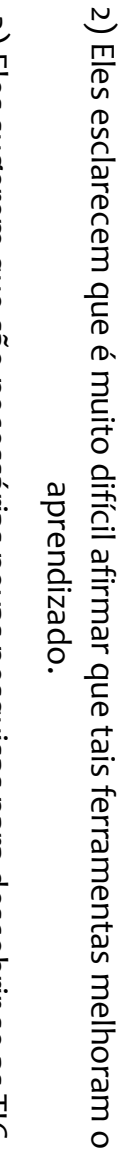 & 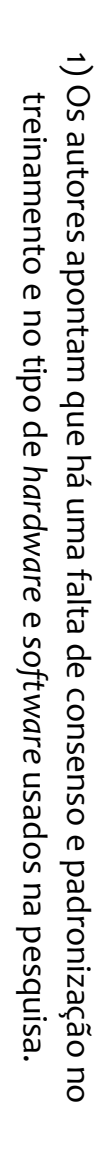 & हू & 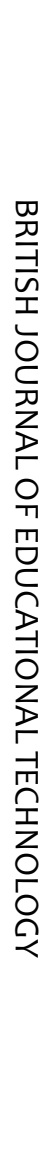 & 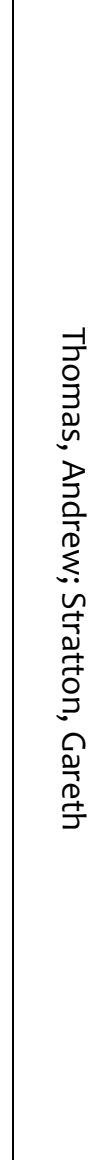 & 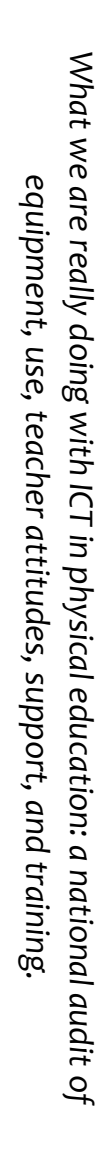 \\
\hline & 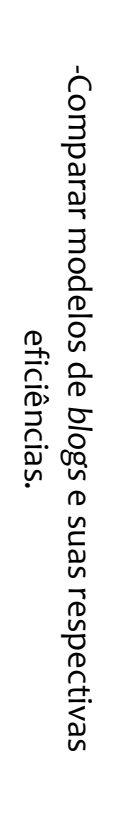 & 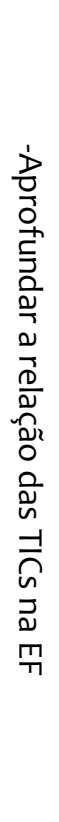 & 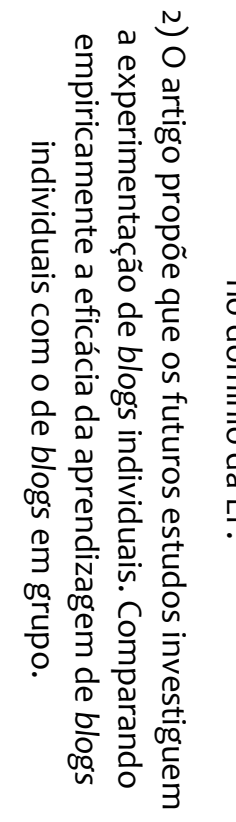 & 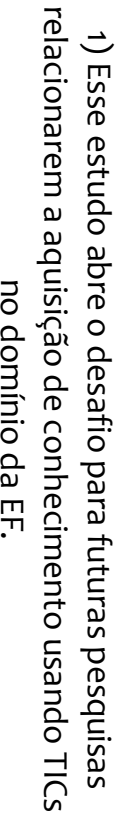 & & & 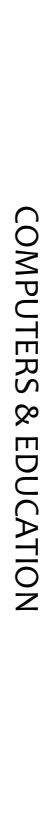 & 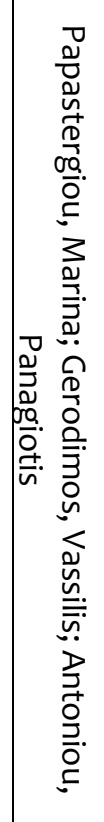 & 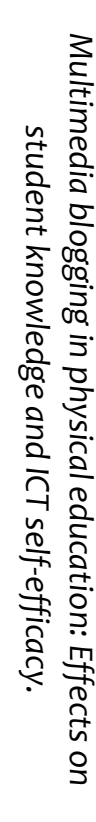 \\
\hline
\end{tabular}




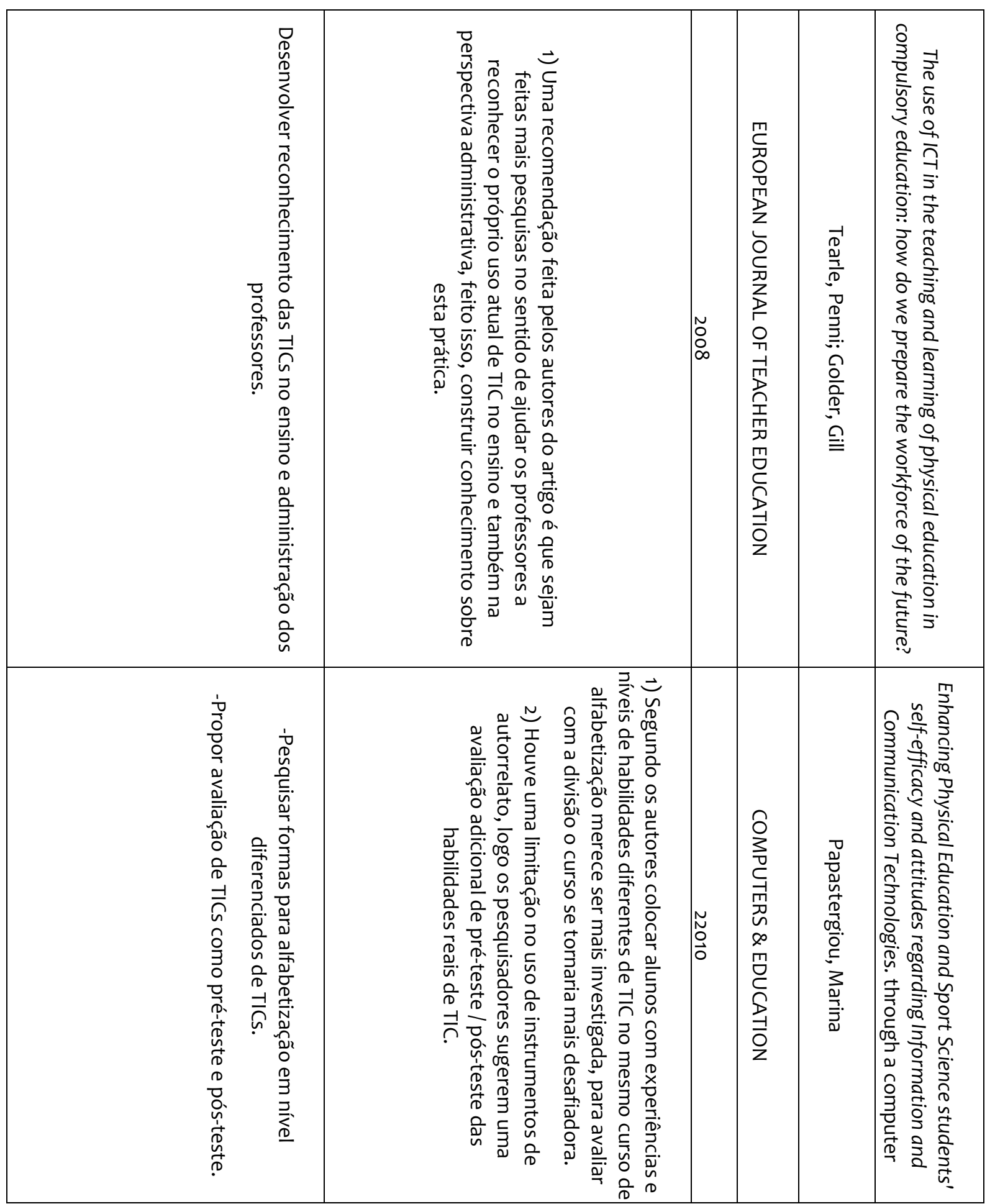




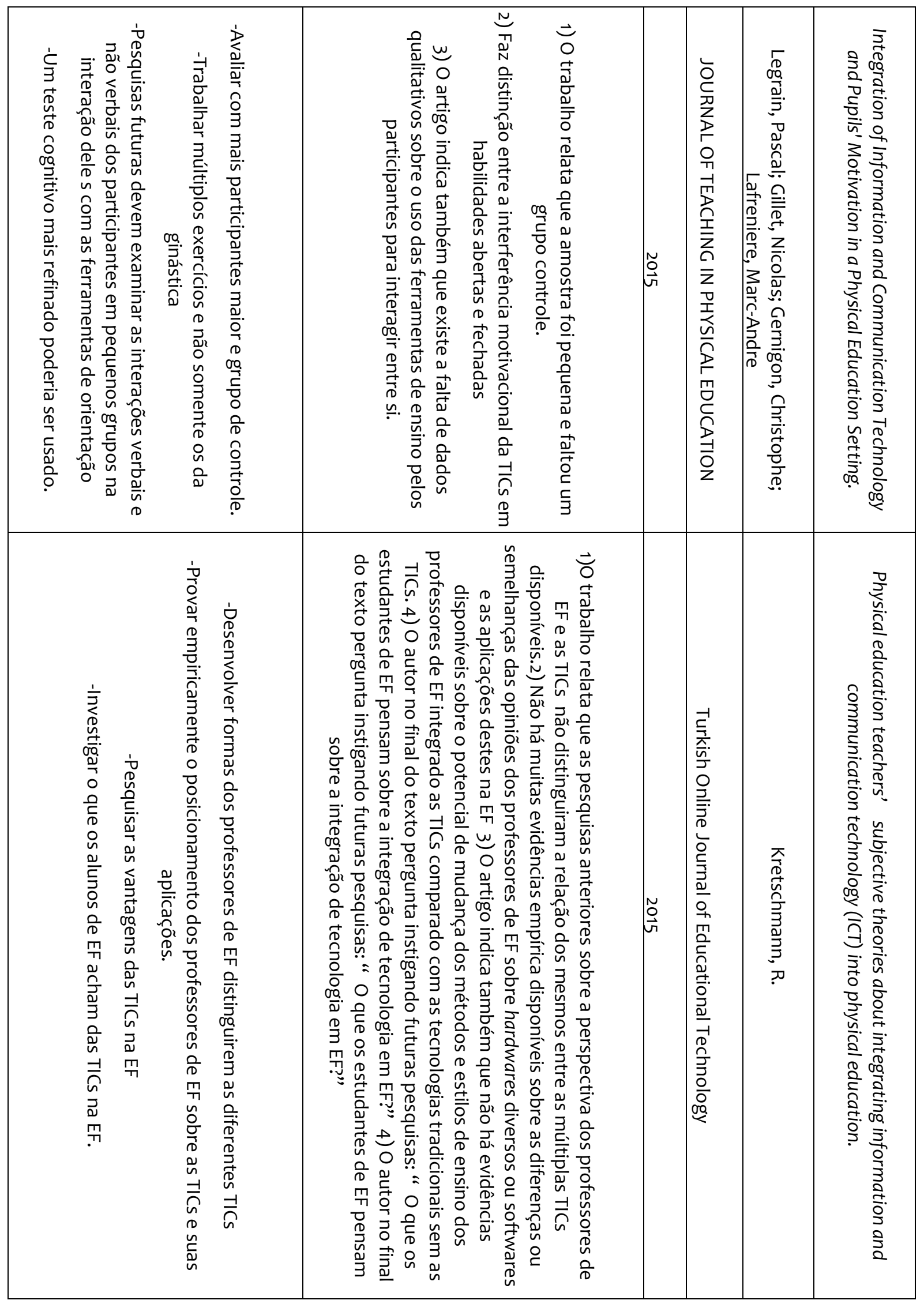

Fonte: Próprio Autor (2020) 
No Quadro 2, foram agrupadas as oportunidades de pesquisa citadas nos artigos em tópicos de semelhança em suas propostas que foram agrupados em 3 tendências:

Relação TICs e EF com um maior foco no(s) aluno(s) "FNA";

Relação TICs e EF com um maior foco no(s) professor(es) "FNP”;

Relação TICs e EF com um maior foco na criação e padronização do(s) material (is) e método(s) “CPMM"

Quadro 2 - Quadro com tendências e oportunidades relacionando os gaps científicos

\begin{tabular}{|c|c|c|c|}
\hline \multicolumn{4}{|c|}{ Tendências e Oportunidades “gaps" } \\
\hline Títulos dos Trabalhos & $\begin{array}{l}\text { Foco no(s) } \\
\text { aluno(s) "FNA" }\end{array}$ & $\begin{array}{l}\text { Foco no(s) } \\
\text { professor(es) } \\
\text { "FNP" }\end{array}$ & $\begin{array}{l}\text { Foco no(s) material } \\
\text { (is) e método (s) } \\
\text { "СРMM" }\end{array}$ \\
\hline $\begin{array}{l}\text { What we are really doing with ICT in } \\
\text { physical education: a national audit } \\
\text { of equipment, use, teacher } \\
\text { attitudes, support, and training. }\end{array}$ & $\begin{array}{l}\text { Pesquisar se as } \\
\text { TICs ajudam a } \\
\text { desenvolver } \\
\text { habilidades } \\
\text { motoras. }\end{array}$ & $\begin{array}{l}\text { Propor modelos } \\
\text { para ministrar aulas } \\
\text { com TICs. }\end{array}$ & $\begin{array}{l}\text { Desenvolver } \\
\text { padronização de } \\
\text { hardwares } \\
\text { softwares. } \\
\text { Pesquisar formas } \\
\text { comprobatórias da } \\
\text { melhora do } \\
\text { aprendizado por } \\
\text { meio das TICs. }\end{array}$ \\
\hline $\begin{array}{l}\text { Multimedia blogging in physical } \\
\text { education: Effects on student } \\
\text { knowledge and ICT self-efficacy. }\end{array}$ & & & $\begin{array}{l}\text { Aprofundar a } \\
\text { relação das TICs na } \\
\text { EF. } \\
\text { Comparar modelos } \\
\text { de blogs e suas } \\
\text { respectivas } \\
\text { eficiências. }\end{array}$ \\
\hline $\begin{array}{l}\text { Enhancing Physical Education and } \\
\text { Sport Science students' self-efficacy } \\
\text { and attitudes regarding Information } \\
\text { and Communication Technologies }\end{array}$ & $\begin{array}{l}\text { Pesquisar formas } \\
\text { para } \\
\text { alfabetização em } \\
\text { nível }\end{array}$ & & $\begin{array}{l}\text { Propor avaliação de } \\
\text { TICs como pré-teste } \\
\text { e pós-teste. }\end{array}$ \\
\hline
\end{tabular}




\begin{tabular}{|c|c|c|c|}
\hline through a computer literacy course. & $\begin{array}{l}\text { diferenciados de } \\
\text { TICS. }\end{array}$ & & \\
\hline $\begin{array}{l}\text { The use of ICT in the teaching and } \\
\text { learning of physical education in } \\
\text { compulsory education: how do we } \\
\text { prepare the workforce of the future? }\end{array}$ & & $\begin{array}{l}\text { Desenvolver } \\
\text { reconhecimento } \\
\text { das TICs no ensino } \\
\text { e administração } \\
\text { dos professores. }\end{array}$ & \\
\hline $\begin{array}{l}\text { Integration of Information and } \\
\text { Communication Technology and } \\
\text { Pupils' Motivation in a Physical } \\
\text { Education Setting. }\end{array}$ & $\begin{array}{l}\text { Pesquisar o } \\
\text { impacto das TICs } \\
\text { nas habilidades } \\
\text { cognitivas e no } \\
\text { desempenho } \\
\text { motor dos alunos }\end{array}$ & & $\begin{array}{l}\text { Estudo testou um } \\
\text { modelo integrativo } \\
\text { sobre o impacto } \\
\text { das } \text { TICs } \\
\text { desempenho no } \\
\text { EF. }\end{array}$ \\
\hline $\begin{array}{l}\text { Physical education teachers' } \\
\text { subjective theories about integrating } \\
\text { information and communication } \\
\text { technology (TIC) into physical } \\
\text { education. }\end{array}$ & $\begin{array}{l}\text { Investigar o que } \\
\text { os alunos de EF } \\
\text { acham das TICs na } \\
\text { EF }\end{array}$ & $\begin{array}{l}\text { Desenvolver } \\
\text { formas dos } \\
\text { professores de EF } \\
\text { distinguirem as } \\
\text { diferentes TICs. } \\
\text { Provar } \\
\text { empiricamente o } \\
\text { posicionamento } \\
\text { dos professores de } \\
\text { EF sobre as TICs e } \\
\text { suas aplicações. }\end{array}$ & $\begin{array}{l}\text { Pesquisar as } \\
\text { vantagens das TICs } \\
\text { na EF }\end{array}$ \\
\hline
\end{tabular}

Fonte: Próprio Autor (2020)

\section{CONSIDERAÇÕES FINAIS}

As três tendências principais para futuras pesquisas relacionando as TICs e a EF foram identificadas, destacando-se sobre elas a ideia de priorizar as novas pesquisas com um foco maior nas relações múltiplas entre as TICs e EF no que diz respeito ao uso, criação e padronização do (s) material (is) e método (s) "CPMM". Isto se dá visto a imensa gama de opções de materiais e métodos, muitas das vezes gerados por conta da 
alta demanda das novas tecnologias, o uso constante e globalizado das webs além de outros fatores que dificultam uma padronização ou treinamento para tais.

Em segundo lugar está a relação TICs e EF com um maior foco no(s) aluno(s) "FNA". Esse tópico pode ser visto em diferentes frentes segundo a análise das oportunidades identificadas nos 6 textos mais citados nos últimos 14 anos. A primeira frente seria aquela na qual as futuras investigações se norteassem para descobrir se as TICs podem de alguma forma auxiliar na aprendizagem motora dos alunos. Possíveis desdobramentos podem advir dessa primeira frente de pesquisa, tal como a busca da relação entre as TICs e o rendimento esportivo, ou com os ensinamentos de jogos e atividades lúdicas, ou então conjecturar a relação das novas tecnologias digitais com as tendências particulares da reabilitação articular e muscular. Ou seja, as possibilidades são muitas.

Essa por fim, uma segunda frente dentro da "FNA" é a aquela que investiga uma perspectiva mais ampla, preocupando-se e inquirindo o que os alunos de EF pensam dessa relação entre as TICs e a EF.

Em terceira instância este estudo relata uma necessidade de se pesquisar a relação TICs e EF com um maior foco no(s) professor(es) "FNP", visto que os mesmos muitas das vezes não percebem ou não conseguem identificar o que é uma TIC, muito menos qual a melhor maneira de usa-la. Além disso, segundo os artigos falta orientação por conta dos gestores e instituições quanto ao tema.

Essa terceira perspectiva ressaltada pelos artigos prevê a oportunidade de estudar diferentes modos de ensinar os alunos em sala de aula, visto que dentro de uma turma, tem-se diferentes alunos com diferentes níveis de conhecimento e habilidades com as TICs. A ideia é que seja proposto uma forma de tornar o aprendizado de TICs desafiador para todos, e tal é de certa forma incumbência dos professores e das instituições.

Este trabalho proporcionou uma contribuição para ciência por meio da identificação dos principais autores e suas obras, que podem servir de base para pesquisas futuras no tema. Além disso, essa pesquisa realizou o mapeamento do estado da arte sobre o assunto, pois fez a sistematização da literatura sobre TICs e EF, O apontamento de autores, journals, países, as publicações nos últimos anos dentro deste campo de estudo. Além disso o estudo também apontou e identificou os gaps científicos 
relatados nos artigos mais citados nas plataformas Web of Science e Scopus, norteando assim propostas para futuras oportunidades de pesquisa.

Como principal contribuição aplicada, este estudo pode auxiliar as práticas operacionais diárias de ensino relacionados às TICs e a EF, ao nortear o acesso à literatura considerada relevante, atualizada e referencial sobre o tema.

A complementação e evolução desta análise também sugere para pesquisas futuras o explorar de novos tópicos que não foram abordados neste trabalho, como por exemplo a continuação da investigação do estado da arte e da evolução do tema em outras bases bibliométricas indexadas.

Um ponto limitante a ser destacado é que muitas das informações obtidas nesse estudo são oriundas de pesquisas escritas sob um outro viés cultural, ou seja, uma perspectiva internacional, as quais muitas das vezes são pautadas em uma diferente cultura educativa, a qual podem diferir dos conceito e informações aplicadas aqui no Brasil.

Esse estudo finda-se com algumas perguntas provocadoras que ajudariam a direcionar essa reflexão: "Quais mudanças a TIC provocou no ensino e na formação dos professores de EF em países que já a utilizam com frequência?”; “Como está a utilização das TICS nas universidades brasileiras?"; "Como as universidades brasileiras estão se preparando para formar futuros professores da área da motricidade humana em vista dessa mudança educacional globalizada e tecnológica?'

\section{REFERÊNCIAS}

ANDERSON, M.; MIKAT, R.; MARINEZ, R. Digital video production in physical education and athletics. Journal of Physical Education, Recreation, and Dance, v. 72, n. 6, aug. 2001, p. 19-21.

ANTONIOU, et al. Applying multimedia computer-assisted instruction to enhance physical education students' knowledge of basketball rules. Physical Education \& Sport Pedagogy, v. 8, n. 1, 2003. P. 78-90.

AUSUBEL, D. P. et al. Educational psychology: a cognitive view. 1968.

BASADRE, R.F; NUNEZ, J. I.H.V.; PATON, R.N. ICT in physical education from the perspective of students in elementary school. Sportis-Scientific Technical Journal Of School Sport Physical Education And Psychomotricity, v. 1, n. 2, 2015, p. 141-155. 
BRASIL. Ministério da Educação (MEC). Decreto n 6.300, de 12 de dezembro de 2007. Dispõe sobre o Programa Nacional de Tecnologia Educacional - Proinfo. Diário Oficial da União, Brasília, DF, 2007.

DAVIS, N.; KEMIS, M.; JOHNSON, N. Path to the future: Generative evaluation for simultaneous renewal of ICT in teacher education and K-12 schools. IFIP Advances in Information and Communication Technology, v. 132, 2003, p. 53-64.

EL TANTAWI, M. Evaluation of a blog used in a dental terminology course for first-year dental students. Journal of Dental Education, v. 72, n. 6, 2008, p. 725-735.

FERREIRA E.R.G; BENDRATH, E.A; BASEI, A,P. Conceptions of the physical education teachers of public schools in a small municipality in the state of Parana, Brasil, About the Use of Information and Communication Technologies. REVISTA EDUCAONLINE, v. 10, n. 1, apr. 2016, p. 75-79.

FERREIRA, M. P. et al. Mergers \& acquisitions research: A bibliometric study of top strategy and international business journals, 1980-2010. Journal of Business Research, v. 67 , n. 12, 2014, p. 2550-2558.

GARCÍA, N.; SÁNCHEZ, S. Incorporación de dispositivos móviles a la educación física escolar. Revista Española de educación física y deportes, n. 5, jun./jul. 2014. p. 79-86.

GOMEZ-JAUREGUI, V. et al. Information management and improvement of citation indices. International Journal of Information Management, v. 34, n. 2, abr. 2014, p. 257271.

GUARDIA, F. J. Las Nuevas Tecnologías, la Educación Física y su integración en el tercer y cuarto nivel de concreción curricular. En actas del XX Congreso Nacional de Educación Física. (CD-ROM ed.). Universidad de Alcalá de Henares, Guadalajara, España, 2002.

HARTVELD, A.; HEGARTY, J. R.; BLURTON, A. Tools to give computer feedback to movement. Physiotherapy, v. 82, set. 1996, p. 509-513.

HIGGINS, S.; MOSELEY, D. Teachers' thinking about information and communications technology and learning: beliefs and outcomes. Teacher Development, v.5, n.2, 2001, p. 191-210.

HUANG, Y. et al. Rehabilitation using virtual reality technology: a bibliometric analysis, 1996-2015. Scientometrics, v. 109, n. 3, 2016, p. 1547-1559.

Information and communication technology. Oxford Reference. Date of access: 17 May. 2019.

From:

http://www.oxfordreference.com/view/10.1093/oi/authority.20110803100002983

JONASSEN, D. H.. Computers as mindtools for schools: engaging critical thinking. Second ed.. Englewood Cliffs, NJ: Prentice Hall, 1999. 
KIRKWOOD, M. et al. Assessment of aerobic endurance: a comparison between CD-ROM and laboratory-based instruction. British Journal of Educational Technology, v. 33, n. 2, 2002, p. 159-172.

MEDEIROS, I. L. DE et al. Revisão sistemática e bibliometria facilitadas por um canvas para visualização de informação. Revista Brasileira de Design da Informação, v. 12, n. 1, 2015, p. 93-110.

MOHNSEN, B. Using technology in physical education. 6th ed. Cerritos, CA: Bonnie's Fitware, 2008.

NIEDERHAUSER, D. S.; STODDART, T. Teachers' instructional perspectives and use of educational software. Teaching and Teacher Education, v. 17, n.1, 2001, p. 15-31.

OLIVEIRA, A. C. A utilização da mídia televisiva no planejamento das aulas de educação física. 2010. 66 p. Monografia de Graduação. Universidade Federal de Minas Gerais. Belo Horizonte - MG. 2010.

PAPASTERGIOU, M. Enhancing physical education and sport science students' selfefficacy and attitudes regarding Information and communication technologies through a computer literacy course. Computers and Education, v. 54, n. 1, 2010, p. 298-308.

PAPASTERGIOU, M.; GERODIMOS, V.; ANTONIOU, P. Multimedia blogging in physical education: effects on student knowledge and ICT self-efficacy. Computers \& Education, v. 57, n. 3, nov. 2011, p. 1998-2010.

PERRENOUD, P. Dez novas competências para ensinar. Porto Alegre: Artmed, 2000.

POLATO, A. Atividades em vídeo nas aulas de educação física. 223 ed. Revista Nova Escola, 2009.

SEMIDÃO R. A. M. Dados, informação e conhecimento enquanto elementos de compreensão do universo conceitual da ciência da informação: contribuições teóricas. Marília, 2014. Dissertação (Mestrado em Ciência da Informação) - Universidade Estadual Paulista, Faculdade de Filosofia e Ciências, 2014.

SMEETS, E. Does ICT contribute to powerful learning environments in primary education?. Computers \& Education, v. 44, n. 3, 1 abr. 2005, p. 343-355.

SUSMAN, E. B. Co-operative learning: a review of factors that increase the effectiveness of computer-based instruction. Journal of Educational Computing Research, v. 18, n. 4, 1998, p. 303-322.

SUZUKI, J.T. F.; RAMPAZZO, S. R. dos R. Tecnologias em educação. São Paulo: Pearson Education do Brasil, 182 p., 2009. 
THOMAS, A.; STRATTON, G. What we are really doing with ICT in physical education: a national audit of equipment, use, teacher attitudes, support, and training. British Journal of Educational Technology, v. 37, n. 4, jul. 2006, p. 617-632.

WIKSTEN, D.; SPANJER, J.; LA MASTER, K. Effective use of multimedia technology in athletic training education. Journal of Athletic Training, v. 37, n. 4, 2002, p. 213-219.

Recebido em: 16/06/2020

Parecer em: 15/10/2020

Aprovado em: 23/02/2020 